\title{
Intraoperative visualization of the pyramidal tract by diffusion-tensor-imaging-based fiber tracking
}

\author{
Christopher Nimsky, ${ }^{\text {a,* }}$ Oliver Ganslandt, ${ }^{\text {a }}$ Dorit Merhof, ${ }^{\text {a }}$ \\ A. Gregory Sorensen, ${ }^{b}$ and Rudolf Fahlbusch ${ }^{\mathrm{a}}$ \\ ${ }^{a}$ Department of Neurosurgery, University Erlangen-Nürnberg, Schwabachanlage 6, 91054 Erlangen, Germany \\ ${ }^{\mathrm{b}}$ Department of Radiology/Nuclear Magnetic Resonance Center, Massachusetts General Hospital, Boston, MA 02129, USA
}

\begin{abstract}
Functional neuronavigation allows intraoperative visualization of cortical eloquent brain areas. Major white matter tracts, such as the pyramidal tract, can be delineated by diffusion-tensor-imaging based fiber tracking. These tractography data were integrated into 3-D datasets applied for neuronavigation by rigid registration of the diffusion images with standard anatomical image data so that their course could be superimposed onto the surgical field during resection of gliomas. Intraoperative high-field magnetic resonance imaging was used to compensate for the effects of brain shift, which amounted up to $8 \mathrm{~mm}$. Despite image distortion of echo planar images, which was identified by non-linear registration techniques, navigation was reliable. In none of the 19 patients new postoperative neurological deficits were encountered. Intraoperative visualization of major white matter tracts allows save resection of gliomas near eloquent brain areas. A possible shifting of the pyramidal tract has to be taken into account after major tumor parts are resected.
\end{abstract}

Keywords: Diffusion tensor imaging; Fiber tracking; Functional neuronavigation; Intraoperative imaging; Major white matter tracts

\section{Introduction}

Integration of functional data from magnetoencephalography (MEG) or functional magnetic resonance imaging (fMRI) has become routine in surgery of tumors adjacent to eloquent cortical regions such as the motor-cortex- or language-related areas (Ganslandt et al., 1999; Kober et al., 2001; Nimsky et al., 1999). This method of so-called functional neuronavigation, however, allows only the identification of functionally critical areas located at the brain surface. If the white matter connections of these

\footnotetext{
* Corresponding author. Fax: +49 91318534551.

E-mail address: nimsky@nch.imed.uni-erlangen.de (C. Nimsky).
}

functional relevant areas are damaged, neurological deficits are inevitable, even if the cortical areas are preserved.

As cortical eloquent brain areas, also major white matter tracts can be identified by intraoperative electrophysiological mapping, such as subcortical electrical stimulation (Duffau et al., 2003; Yingling et al., 1999). However, positive identification of the pyramidal tract by direct stimulation may indicate that the resection has already reached the pyramidal tract or its immediate vicinity so that the resection might have been too extensive, resulting in postoperative deficits. In contrast to invasive mapping, imaging-based localization of functional areas has the advantage that the information on functional localization is already available before surgery, allowing preoperative planning. These data can then be integrated into navigational datasets for intraoperative guidance.

Differences in anisotropy of white and gray matter depicted by diffusion-weighted imaging (Basser et al., 1994) could be applied for localizing the pyramidal tract (Inoue et al., 1999). These data were not only used for preoperative planning, but also for intraoperative guidance, to identify the pyramidal tract during brain tumor resection (Coenen et al., 2001; Kamada et al., 2003).

Diffusion tensor imaging (DTI) can resolve the dominant fiber orientation in each voxel element. The direction of greatest diffusion measured by DTI parallels the dominant orientation of the tissue structure in each voxel, representing the mean longitudinal direction of axons in white matter tracts (Le Bihan and Van Zijl, 2002). DTI provides information about the normal course, the displacement, or interruption of white matter tracts around a tumor, as well as a widening of fiber bundles due to edema or tumor infiltration can be detected (Beppu et al., 2003; Clark et al., 2003; Hendler et al., 2003; Lu et al., 2003; Price et al., 2003; Tummala et al., 2003; Wieshmann et al., 1999; Witwer et al., 2002; Yamada et al., 2003). Integration of these DTI data into navigational setups, e.g., by registering fractional anisotropy maps with 3-D anatomical data, was the next logical step for intraoperative localization of major white matter tracts (Nimsky et al., 2005c; Talos et al., 2003; Wu et al., 2003). 
Parallel to these developments, various fiber tracking algorithms which compare local tensor field orientations measured by DTI from voxel to voxel have been implemented, allowing a noninvasive tracing of large fiber tract bundles in the human brain (Basser et al., 2000; Mori et al., 1999; Mori and Van Zijl, 2002; Stieltjes et al., 2001). Recently, fiber tracking has been used for preoperative visualization of white matter tracts in patients with space occupying lesions (Clark et al., 2003; Hendler et al., 2003; Holodny et al., 2001). Integrating fiber tract data in neuronavigational setups to compare the site of electrical stimulation with the position of the pyramidal tract was demonstrated recently with first preliminary data (Kamada et al., 2005; Kinoshita et al., 2005).

The aim of this study was to investigate how tractography data based on DTI could be integrated into a navigational 3-D dataset and used for intraoperative visualization of the pyramidal tract in glioma surgery.

\section{Materials and methods}

\section{Patient population}

Nineteen patients ( 8 female, 11 male, age range: $21-66$ years; mean: $43.6 \pm 13.3$ years) with supratentorial gliomas (World Health Organization (WHO) grading: I: 1, II: 2, III: 10, IV: 6) which all had a certain spatial relation to the pyramidal tract, i.e., the tumor at least displaced the pyramidal tract, were investigated. This included 17 craniotomies and 2 burr hole biopsies. In the 2 burr hole cases, only a biopsy was performed due to a distinct infiltration of eloquent brain areas. In 7 patients $(4,8,9,11,12$ $14,16)$, a recurrent tumor was operated on. The local ethical committee of the University Erlangen-Nuremberg approved intraoperative high-field MRI in combination with neuronavigation, and signed informed consent was provided by each patient. Patient details are listed in Table 1.

\section{Pre- and intraoperative imaging}

Pre- and intraoperative imaging is performed with a $1.5 \mathrm{~T}$ Magnetom Sonata Maestro Class scanner (Siemens AG Medical Solutions, Erlangen, Germany) placed in an operating room with radiofrequency (RF) shielding. This is a high-field scanner with a superconductive $1.5 \mathrm{~T}$ magnet with a length of $160 \mathrm{~cm}$ and an inner bore diameter of $60 \mathrm{~cm}$ equipped with gradient system with a field strength of up to $40 \mathrm{mT} / \mathrm{m}$ (effective $69 \mathrm{mT} / \mathrm{m}$ ) and a slew rate of up to $200 \mathrm{~T} / \mathrm{m} / \mathrm{s}$ (346 T/m/s effective). The patient is placed on a rotatable surgical MR-compatible tabletop (Trumpf, Saalfeld, Germany) which is adapted to the scanner. The principal surgical position is at $160^{\circ}$, with the patient's head placed at the $5 \mathrm{G}$ line in an MR-compatible headholder that is integrated into an adapted head coil. For scanning, the table is turned $160^{\circ}$ into the scanner. For anesthesia, MR-compatible ventilation and monitoring equipment is used (Nimsky et al., 2004).

Preoperative imaging on the days before surgery consisted of standard anatomical imaging applying routine $\mathrm{T} 1$ - and $\mathrm{T} 2$ weighted sequences to visualize tumor anatomy, DTI acquisition for tractography, and fMRI measurements in the patients with a tumor adjacent to the central region. Preoperative tractography and fMRI data were integrated into 3-D T1-weighted data as described below. A further 3-D anatomical dataset was acquired preoperatively after induction of anesthesia and head fixation for patient registration (see Neuronavigation section). Intraoperative imaging included standard T1- and T2-weighted image data (identical to the preoperative sequences), as well as a DTI acquisition for intraoperative tractography. The intraoperative tractography data were used to visualize the position and configuration of the pyramidal tract and were displayed during surgery along the intraoperative diffusion images so that the neurosurgeon could evaluate the actual intraoperative relation of the shifted pyramidal tract to the resection cavity or some

Table 1

Nineteen patients with supratentorial gliomas undergoing surgery with intraoperative visualization of the pyramidal tract

\begin{tabular}{|c|c|c|c|c|c|c|c|}
\hline Pat No. & Age, sex & Location & $\begin{array}{l}\text { WHO } \\
\text { grading }\end{array}$ & $\begin{array}{l}\text { TRE } \\
{[\mathrm{mm}]}\end{array}$ & fMRI & Intraoperative MRI & $\begin{array}{l}\text { Shift } \\
{[\mathrm{mm}]}\end{array}$ \\
\hline 1 & $48, \mathrm{f}$ & lt postcentral & I & 0.9 & - & Correct biopsy confirmed & 0 \\
\hline 2 & $35, \mathrm{f}$ & lt precentral & III & 1.7 & + & Resection extended (gross total) & -2 \\
\hline 3 & $51, \mathrm{~m}$ & lt postcentral & III & 2.1 & + & Resection extended (subtotal) & -2 \\
\hline 4 & $44, \mathrm{~m}$ & rt precentral & III & 1.7 & + & Resection extended (subtotal) & -2 \\
\hline 5 & $66, \mathrm{~m}$ & rt temporal & III & 0.5 & + & Resection extended (subtotal) & -6 \\
\hline 6 & $45, \mathrm{~m}$ & rt temporal & IV & 1.2 & - & Gross total resection confirmed & -3 \\
\hline 7 & $52, \mathrm{~m}$ & lt postcentral & III & 0.4 & + & Correct biopsy confirmed & 0 \\
\hline 8 & $32, \mathrm{f}$ & lt postcentral & III & 0.3 & + & Subtotal resection confirmed & -3 \\
\hline 9 & $30, \mathrm{f}$ & lt precentral & IV & 0.9 & + & Subtotal resection confirmed & -2 \\
\hline 10 & $37, \mathrm{f}$ & lt precentral & III & 0.5 & + & Subtotal resection confirmed & +2 \\
\hline 11 & $21, \mathrm{~m}$ & rt postcentral & II & 1.5 & - & Subtotal resection confirmed & +8 \\
\hline 12 & $29, \mathrm{f}$ & rt precentral & II & 1.0 & + & Subtotal resection confirmed & -2 \\
\hline 13 & $29, \mathrm{~m}$ & rt precentral & III & 1.3 & + & Gross total resection confirmed & -2 \\
\hline 14 & $64, \mathrm{~m}$ & re postcentral & IV & 0.6 & + & Resection extended (gross total) & -4 \\
\hline 15 & $39, \mathrm{~m}$ & lt temporal & III & 0.4 & + & Resection extended (subtotal) & +5 \\
\hline 16 & $33, \mathrm{f}$ & rt precentral & III & 1.4 & + & Subtotal resection confirmed & +1 \\
\hline 17 & $55, \mathrm{f}$ & lt precentral & IV & 0.9 & + & Gross total resection confirmed & 0 \\
\hline 18 & $60, \mathrm{~m}$ & rt precentral & IV & 0.8 & + & Gross total resection confirmed & +6 \\
\hline 19 & $59, \mathrm{~m}$ & rt temporal & IV & 1.0 & - & Subtotal resection confirmed & -5 \\
\hline
\end{tabular}

TRE: target registration error as measure for patient registration accuracy; fMRI: functional magnetic resonance co-registered to fiber tract data; intraoperative MRI: results and consequences of intraoperative MRI; shift: extent of shifting of the pyramidal tract (positive values indicate outward, negative values inward movement). 
remaining tumor. Furthermore, these intraoperative data were integrated into the navigational dataset after surgery to measure the extent of white matter tract shifting.

\section{Neuronavigation}

Microscope-based neuronavigation support is provided by the VectorVision Sky navigation system (BrainLab, Heimstetten, Germany), which is integrated into a radiofrequency-shielded operating theater that is designed for intraoperative MRI (Nimsky et al., 2004). An $1.0 \mathrm{~mm}$ isotropic 3-D MPRAGE dataset (magnetization prepared rapid acquisition gradient echo sequence; TE $4.38 \mathrm{~ms}$, TR $2020 \mathrm{~ms}$, matrix size $256 \times 256$, FOV $250 \mathrm{~mm}$, slice thickness $1 \mathrm{~mm}$, slab $16 \mathrm{~cm}$, measurement time 8 min $39 \mathrm{~s}$ ) was acquired after head fixation and induction of anesthesia prior to surgery as navigational reference dataset, in which functional data could be integrated. For registration, 5 adhesive skin fiducials were placed in a scattered pattern on the head surface prior to imaging and registered with a pointer after their position was defined in the 3-D dataset. As estimate for the clinical navigation accuracy, the target registration error localizing a separate fiducial, which was not used for registration, was documented. Furthermore, repeated landmark checks were performed during surgery to ensure overall ongoing clinical application accuracy.

\section{Integration of $f M R I$}

Functional data from fMRI, that were acquired preoperatively, were integrated into the navigational 3-D dataset in patients with lesions adjacent to the motor cortex. For fMRI, we applied an echo planar imaging sequence (TE $60 \mathrm{~ms}$, TR $1580 \mathrm{~ms}$, matrix size $64 \times 64$, FOV $192 \mathrm{~mm}$, slice thickness $3 \mathrm{~mm}, 16$ slices, measurement time: 3 min $13 \mathrm{~s}$ ). The stimulation was done in a block paradigm ("boxcar" design) with 120 measurements in 6 blocks. Twenty acquisitions rest were alternating with 20 acquisitions activation in which the patient was instructed to perform fast movements with the limb under investigation. For motion correction, we applied an image-based prospective acquisition correction applying interpolation in the k-space. For evaluation, we used the BrainVoyager 2000 software (version 4.9.2.0, Brain Innovation B.V., Maastricht, The Netherlands). Activation maps were determined by analyzing the correlation for each voxel between signal intensity and a square wave reference function according to the paradigm. Voxels exceeding a significance threshold (typically correlations above a threshold of 0.44 ) were displayed if at least 6 contiguous voxels built a cluster to eliminate isolated voxels. The functional slices were aligned to MPRAGE images with 160 slices obtained in the same patient position.

Various different imaging sequences were registered using the ImageFusion Software (iPlan 1.0, BrainLab, Heimstetten, Germany), performing a semi-automatic rigid registration. After a rough user-dependent alignment, the images are registered using a rigid registration algorithm applying an intensity-based pyramidal approach using mutual information (Studholme et al., 1996; Thevenaz and Unser, 1996). To confirm the fMRI-based identification of the motor cortex, we additionally applied phase reversal of somatosensory-evoked potentials placing a strip electrode (4 contacts) over the presumed central sulcus (Romstöck et al., 2002).
We applied a single-shot spin-echo diffusion-weighted echo planar imaging sequence (TE $86 \mathrm{~ms}$, TR $9200 \mathrm{~ms}$, matrix size $128 \times 128$, FOV $240 \mathrm{~mm}$, slice thickness $1.9 \mathrm{~mm}$, bandwidth $1502 \mathrm{~Hz} / \mathrm{Px}$, using $b$ values of 0 and $1000 \mathrm{~s} / \mathrm{mm}^{2}, 60$ slices, no intersection gap, measurement time $5 \mathrm{~min} 31 \mathrm{~s}$ at 5 averages) for DTI. This sequence is based on a balanced diffusion gradient design which strongly minimizes eddy-current artifacts compared to a single-refocused design. One image without diffusion weighting $\left(b=0 \mathrm{~s} / \mathrm{mm}^{2}\right)$ and six diffusion-weighted images were obtained with the diffusion-encoding gradients directed along the following axes $( \pm 1,1,0),( \pm 1,0,1)$, and $(0,1, \pm 1)$.

\section{Fiber tracking}

The tractography method employed was similar to that of Basser and Mori (Basser et al., 2000; Mori and Van Zij1, 2002; Mori et al., 1999; Stieltjes et al., 2001). Fiber tracts were calculated using the DTI task card version 1.6× (Magnetic Resonance Center, Massachusetts General Hospital, Boston) software on a Siemens scanner satellite console using MR software MRease N4_VA21B under syngo VB10I. We applied a knowledge-based dual-ROI (region of interest) approach, where the tracking algorithm was initiated from user-defined seed regions. Tracking was initiated in both retrograde and orthograde directions according to the direction of the principal eigenvector in each voxel of the ROI. Starting from the seed point, which could be anywhere in the brain space, the tensor information (eigenvalues and eigenvectors) at the seed point is calculated by interpolating tensor data. The track point moves along the direction of the principle eigenvector for a distance of the step length (predefined, usually $1 / 3-1 / 4$ of voxel size). Then, the new tensor information at that interpolated point is calculated. The track moves along the new direction for another step length, and then new tensor values are calculated. At each step, the fractional anisotropy (FA) and $b=0$ value are calculated by interpolation. The angle between the current and the previous directions is also calculated. If any of these values does not meet the pre-defined threshold, the tracking will stop. At each seed point, the tracking algorithm is applied twice along opposite directions so that tracks from both sides of the seed point can be calculated. If two seed regions are applied for fiber tracking, only fibers passing through both seed regions are visualized. One seed region was placed in the precentral gyrus; in case the precentral gyrus was identified by fMRI (see above), the fMRI data were used as marker for the precentral gyrus which was then taken as a whole as seed region. The second seed region was placed in the posterior parts and the genu of the internal capsule between the thalamus and the lentiforme nucleus identified in colorencoded FA maps where the blue color indicated the cranio-caudal direction of the fibers. A vector step lengths of $0.5 \mathrm{~mm}$, an angular threshold of $35^{\circ}$, and an FA threshold of 0.3 were used in this study. The tractography results were also assigned color applying the convention used in the color-encoded fractional anisotropy maps. The color mapping scheme defines white matter tracts oriented in an anterior-posterior direction in green, a left-right direction in red, and a superior-inferior direction in blue, assuming the patient is lying in supine position and the head is not tilted (Pajevic and Pierpaoli, 1999).

The generation of the fiber tracts and visualization along with the diffusion data lasted only about 1-2 min so that this technique could also be applied intraoperatively. 


\section{Integrating tractography data into navigation}

The tractography results were saved in a plain text file containing the $x / y / z$ coordinates of each fiber. These data were imported along with the $b=0$ diffusion images into the navigation planning software (import module for iPlan 1.0 programmed by $\mathrm{U}$. Mezger, BrainLab, Heimstetten, Germany). After rigid registration of the $b=0$ images with the standard 3-D anatomical images, the fiber tracts could be displayed in the standard anatomical images. Then, their outline was segmented by thresholding so that the whole fiber tract bundle could be defined as an object in the navigation system and displayed during surgery.

The whole process of preoperative processing of DTI data for integration into the navigation system, including fiber tract generation, import into the navigation system, registration with the 3-D dataset, and the final segmentation for 3-D object generation, lasted about $20 \mathrm{~min}$ in each case.

\section{Non-linear registration of $b=0$ diffusion images and anatomical image data}

For estimation of the spatial error caused by the rigid registration of DTI data with 3-D anatomical datasets which does account for the image distortion of the echo planar imaging technique of DTI, we performed a non-linear registration technique based on Bézier functions. This non-linear registration was so time-consuming (needing several hours in each case) that it could not be applied in the preoperative clinical routine setting so that all data were evaluated postoperatively.

In a first step, the $b=0$ dataset was rigidly registered to the MPRAGE dataset. The result of the rigid registration was taken as an initial estimation for a non-rigid transformation which was then performed using Free-Form Deformation (FFD). To accomplish the deformation graphics, hardware-based three-dimensional Bézier functions were considered since Bézier functions provide a mechanism for their modification and are characterized by intuitive behavior on their change. The main idea of the non-rigid registration is to manipulate free control points in the lattice in such a way that the similarity measure between the $b=0$ volume which is deformed with FFD and the reference MPRAGE volume takes its maximum (Merhof et al., 2004). Thus, the registration task is equivalent to a multidimensional optimization problem. The quality measure which was used to assess the quality of the alignment is based on normalized mutual information.

The accuracy of registration of the $b=0$ images, defining the coordinate system for the fiber tracts, with the 3-D anatomical dataset was monitored by a semi-transparent overlay display and by displaying one dataset as an insert in the other one.

\section{Evaluation of white matter tract shifting}

Pre- and intraoperative $b=0$ diffusion images along with the MPRAGE sequences were registered using the ImageFusion Software (iPlan 1.2, BrainLab, Heimstetten, Germany) to measure the extent of maximum white matter tract shifting. Then, a semiautomatic rigid registration is applied, that is, after a rough alignment by the user, the images are registered by the software using a rigid registration algorithm applying an intensity-based pyramidal approach using mutual information (Studholme et al., 1996; Thevenaz and Unser, 1996). To evaluate the maximum extent of shifting, the outer borders of the reconstructed pyramidal tracts of the pre- and intraoperative data were segmented (see above), and then the maximum distance between the corresponding pre- and intraoperative contours was measured. According to the direction of shifting, which was referred to the craniotomy opening, positive or negative values were assigned: positive for a movement towards the surface (i.e., swelling), negative for inward movements.

\section{Results}

Fiber tracking

The fiber tract bundle representing the pyramidal tract could be generated in all patients using both the pre- and intraoperative acquired DTI data. The fiber tract visualization along with the diffusion images displayed at standard projections allowed a direct representation of the relation of the pyramidal tract to the tumor regarding the preoperative (Fig. 1), as well as the intraoperative data.

Considering the situation after intraoperative imaging, the intraoperative image data could be used for an immediate visualization of the shifted pyramidal tract in relation to the resection cavity or some remaining tumor so that the neurosurgeon could decide whether to continue the resection or not. In 6 of the 19 patients $(31.6 \%)$, the resection was extended due to intraoperative imaging with updated anatomical image data (see Table 1). In this navigation update, the generated fiber tracts based on intraoperative DTI data were not integrated into the navigational update because of the time constraints during surgery because the whole integration process would have resulted in about $20 \mathrm{~min}$ additional intraoperative waiting time.

\section{Integration of fiber tract data into anatomical images}

Integration of the preoperative fiber tract data into the 3-D anatomical navigational dataset was technically feasible in all patients (Figs. 1 and 2). In 15 patients, data from fMRI identifying the precentral gyrus were co-registered, identifying the motor gyrus as seed region for the fiber tracking algorithm. Accordingly, the localization of fMRI and DTI showed a good congruency, that is, the volume assigned to the pyramidal tract started in close proximity to the gyrus, which was identified as the precentral gyrus by motor fMRI.

\section{Registration accuracy and image distortion}

In the area of interest, i.e., the pyramidal tract adjacent to the tumor, there was a good correspondence between $b=0$ images and the 3-D dataset with a deviation of less than $3 \mathrm{~mm}$ (Fig. 3). In the slices of the upper cortical surface and the lower brainstem, anatomical distortions of the $b=0$ images due to susceptibility artifacts of echo planar imaging technique revealed larger deviations. In case of a tumor reaching the cortical surface near the central region, the application of the rigid registration algorithm for definition of the fiber tract coordinate system could be narrowed to that region so that the registration error in this area of interest could also be below $3 \mathrm{~mm}$.

The analysis of the distortion of the diffusion images by the non-linear registration approach revealed an image distortion below $2 \mathrm{~mm}$ in the left-right direction and a more varying image 

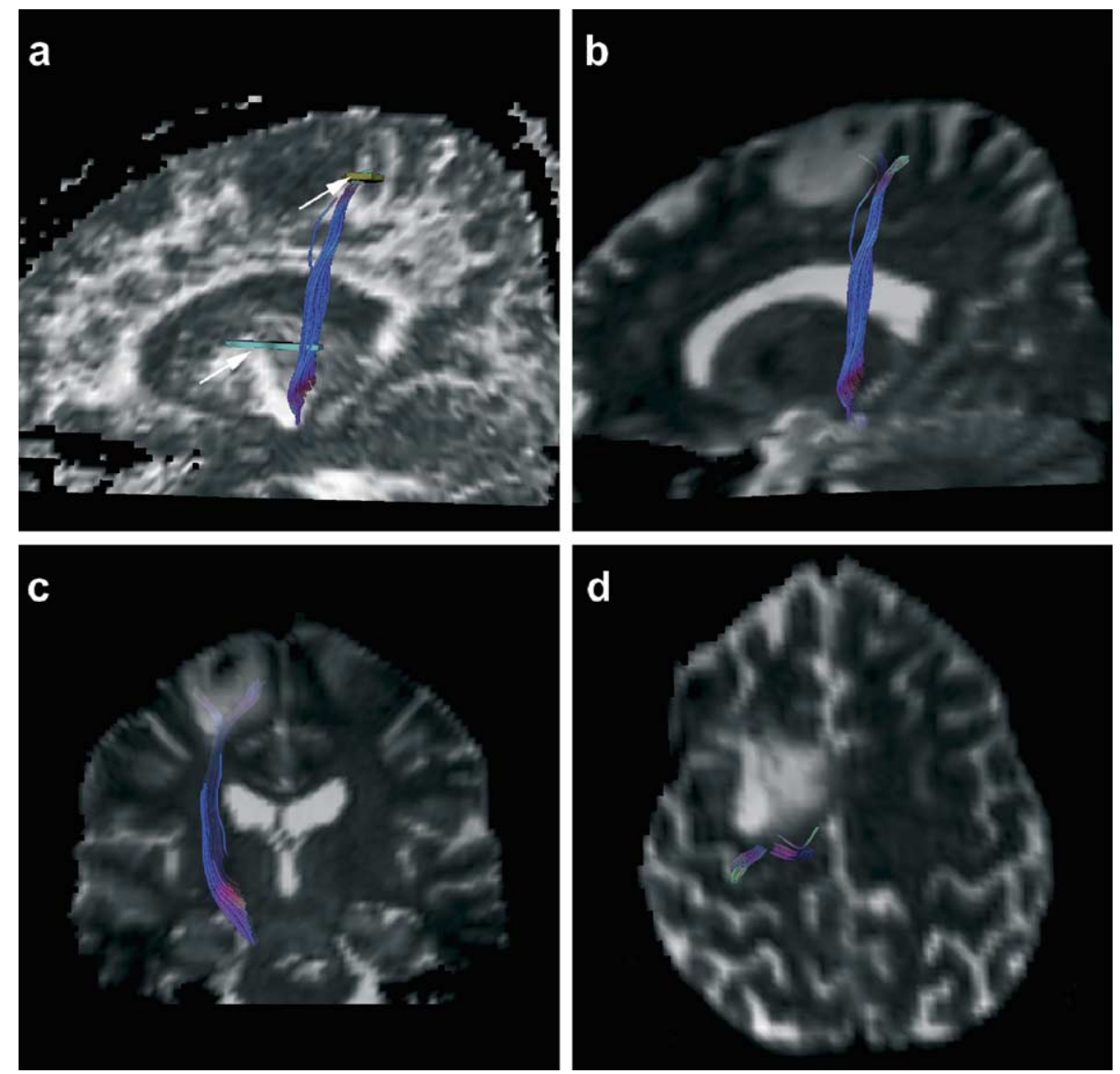

Fig. 1. Fiber tracking in a patient with a recurrent right precentral WHO grade II astrocytoma (patient no. 12); the tumor is directly adjacent to the pyramidal tract. (a) Right pyramidal tract with the two region of interests (ROIs) applied as user-defined seed regions for the tracking algorithm (white arrows: brown ROI in the motor gyrus; green ROI in the internal capsule) displayed along with the sagittal plane of the fractional anisotropy map. (b-d) Display of the pyramidal tract along with the $b=0$ images: (b) Sagittal plane of the $b=0$ image. (c) Transparent display of the coronal $b=0$ image. (d) Non-transparent display of the axial $b=0$ image.
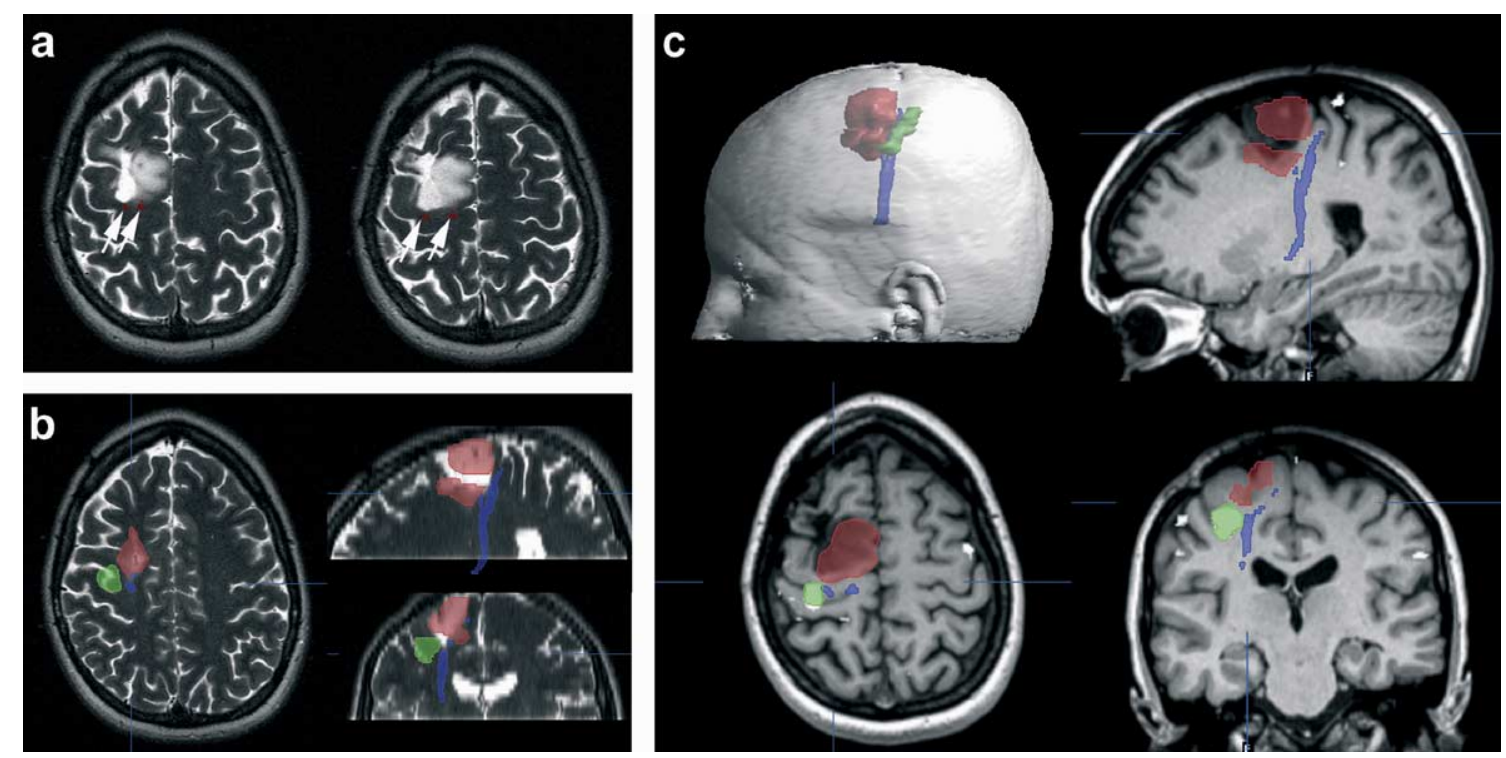

Fig. 2. Generation of a 3-D object representing the whole fiber tract bundle of a pyramidal tract. Same patient as in Fig. 1. (a) Axial T2-weighted images after definition of the coordinate system of the fiber tracts by rigid registration with the $b=0$ diffusion images so that the fiber tract data are displayed in the standard anatomical images (white arrows). (b) After segmentation of the fiber tract bundle, a 3-D object (blue) is generated representing the right pyramidal tract; motor fMRI is segmented in green, the tumor in red. (c) Same display as in Fig. 2b, instead of the T2-weighted images, the isotropic T1-weighted images are displayed in axial, coronal, and sagittal plane, as well as a 3-D rendering, depicting the three objects (tumor and fMRI and pyramidal tract). 


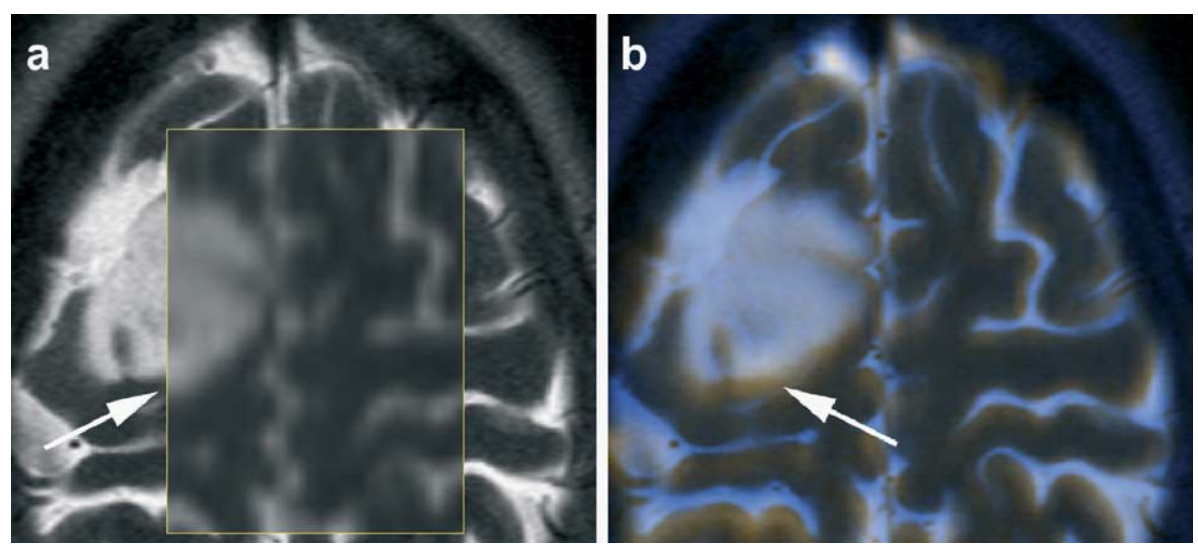

Fig. 3. Registration of distorted $b=0$ diffusion images with standard anatomical image data to define the coordinate system of the fiber tracts. The registration error was below $3 \mathrm{~mm}$ (same patient as in Figs. 1 and 2). (a) Application of the magic lens; in the yellow rectangle, the registered $b=0$ image is displayed, outside the rectangle, a standard T2-weighted image is shown; the white arrow depicts the registration error in anterior-posterior direction. (b) The same slice in a transparent overlay mode, mismatching areas are delineated in yellow (white arrow).

distortion in the phase-encoding (anterior-posterior) direction. This anterior-posterior distortion had its maximum in the cortical area and in the area of the lower brain stem due to susceptibility artifacts of the echo planar images. Summarizing the amount of anterior-posterior distortion revealed that, in a region of $6.8 \mathrm{~cm}$ (36 slices) starting $2 \mathrm{~cm}$ below the cortical surface, the mean image distortion was below $3 \mathrm{~mm}$. Except for an extension to the cortical surface, in this region of low image distortion, all tumors were localized so that the image registration error corresponded well to the extent of image distortion.

\section{Intraoperative visualization in the surgical field}

The clinical application accuracy of the navigation measured as target registration error was in the range of 0.3 to $2.1 \mathrm{~mm}$ (mean \pm standard deviation: $1.0 \pm 0.5 \mathrm{~mm}$ ). The outline of the pyramidal tract could be displayed in the microscope field of view by the microscope heads-up display along with the tumor contour in the 17 craniotomy cases (Fig. 4). In the 2 burr hole cases, the localization of the pyramidal tract was displayed in the navigational dataset when the biopsy trajectory was planned and during the actual biopsy when the biopsy canula was tracked, and its actual position was delineated on the navigation screen.

The intraoperative visualization of the course of the pyramidal tract besides the tumor contour facilitated preservation of eloquent brain structures. The display of contours in the surgical field gave a very intuitive 3-D impression, how the course of the pyramidal tract was in the actual situation so that the surgeons were able to maximize the resection without increasing morbidity. Both surgeons (C.N. and R.F.) agreed that without this information they would have been less aggressive in their resections.

The result of preoperative non-invasive mapping of the motor gyrus by fMRI and co-registered fiber tracking was confirmed by phase reversal of somatosensory-evoked potentials in the 14 patients undergoing craniotomy in the area of the precentral gyrus so that a strip electrode could be placed over the central sulcus (Fig. 4b).

In 4 of the 19 patients, there was a preoperative paresis, which resolved in 2 of them preoperatively after treatment with dexamethasone. Among the 2 patients with a distinct preoperative paresis in one (patient no. 7), the paresis did not change, and, in the other patient (no. 9), the paresis improved after surgery. We did not encounter new neurological deficits in the postoperative course of the patients.

\section{Intraoperative imaging and brain shifting}

Intraoperative MRI revealed a shifting of the pyramidal tract that ranged from -6 to $+8 \mathrm{~mm}$ (mean \pm standard deviation: $-0.6 \pm$ $3.7 \mathrm{~mm}$ ). In 3 patients, no shifting was detected, in 11, an inward movement (assigned with negative values; mean \pm standard deviation: $-3.0 \pm 1.4 \mathrm{~mm}$ ) (Fig. 5), and in 5, an outward movement was detected (mean \pm standard deviation: $+4.4 \pm 2.9$ $\mathrm{mm})$.

\section{Discussion}

The concept of integrating functional data from MEG and fMRI into anatomical 3-D datasets has been widely used for preoperative neurosurgical planning, as well as for intraoperative identification of eloquent structures, resulting in so-called functional neuronavigation (Ganslandt et al., 1999; Nimsky et al., 1999). In this study, we have demonstrated that it is possible to integrate fiber tract data from DTI, representing major white matter tracts, into the navigational dataset as well. This approach allowed visualizing the course of the pyramidal tract during surgery. This intraoperative knowledge about the localization of eloquent brain areas allows safe resections of tumors adjacent to deep-seated eloquent brain areas. Fortunately, none of our patients encountered a new postoperative deficit.

New in the demonstrated approach is that it is possible to integrate fiber tract bundles, which are generated by fiber tracking algorithms, into navigational datasets directly. Up to now, except for some recent feasibility studies (Kamada et al., 2005; Kinoshita et al., 2005; Talos et al., 2003), integration of the fiber tract information into common 3-D navigational datasets has not yet been routinely implemented. Compared to previous attempts based on diffusion-weighted images (Coenen et al., 2001; Kamada et al., 2003) and manual user-dependent and time-consuming segmentation of individual axial slices (Nimsky et al., 2005c; Wu et al., 2003), the direct integration of fiber tract data is much more straightforward and objective, as well as more applicable in a routine clinical setting. 

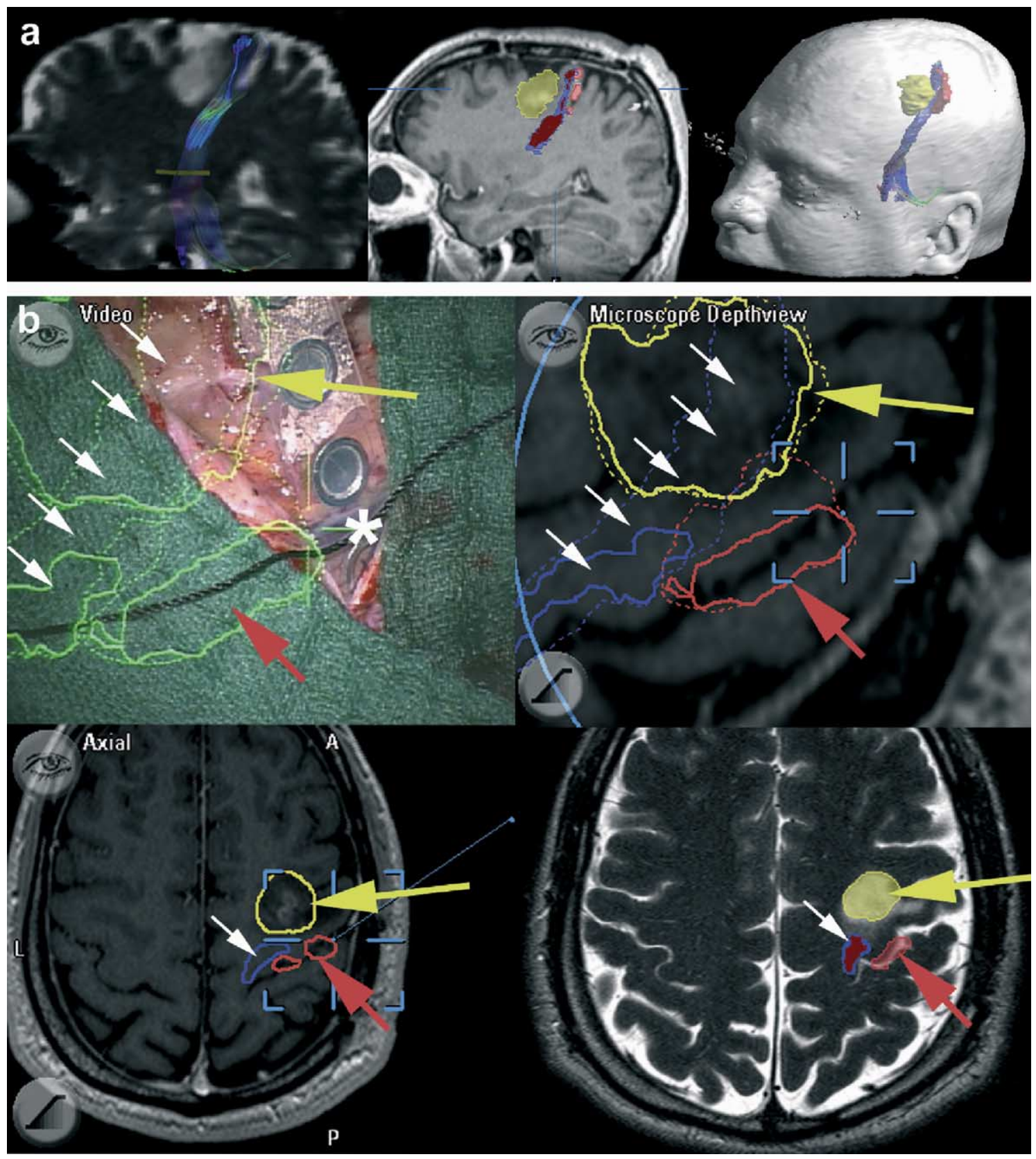

Fig. 4. Intraoperative visualization of the right pyramidal tract in a 44-year-old male patient (no. 4) with a recurrent right precentral WHO grade III astrocytoma. (a) Overview how the 3-D object of the pyramidal tract is generated: left: $b=0$ image with fiber tracking; middle: sagittal view with registered T1-weighted anatomical dataset and segmented tumor (yellow), fMRI (red), and pyramidal tract (blue outline with red inserts representing the fibers after integration in the 3-D dataset); right: 3-D rendering. (b) Intraoperative view: upper part shows microscope video (all contours projected on the surgical field are in green color) and corresponding oblique T1-weighted image with the same contours (yellow arrow: tumor contour; red arrow: motor fMRI marker; white arrows: course of the pyramidal tract (contour in blue); white star: electrode strip for phase reversal of somatosensory-evoked potentials for confirmation of fMRI; the black silk suture placed on the surgical field represents the course of the central sulcus depicted by fMRI and phase reversal)—note that the injected green contours in the microscope video are outlined if the object is in the focal plane, they are dotted if the object is below the focal plane depicting the course of the pyramidal tract in the depth. Lower part: T1- and T2-weighted axial images showing the corresponding structures (note: for better comparison to the intraoperative view, they are depicted as viewed from above).

In fiber tracking, the only user-dependent steps are the definition of seed regions and the choice of tracking parameters. fMRI data can serve as objective seed regions, further reducing the user-dependent bias (Guye et al., 2003; Hendler et al., 2003; Krings et al., 2001). Another advantage of the implemented fiber tracking integration is that the technique applying segmentation in FA maps gets quite imprecise close to the cortical surface. Furthermore, white matter tracts, such as the optic radiation, fibers connecting the language areas Broca and Wernicke, or connections of the fornix to the temporo-mesial lobe cannot be reliably extracted from FA maps without fiber tracking. 

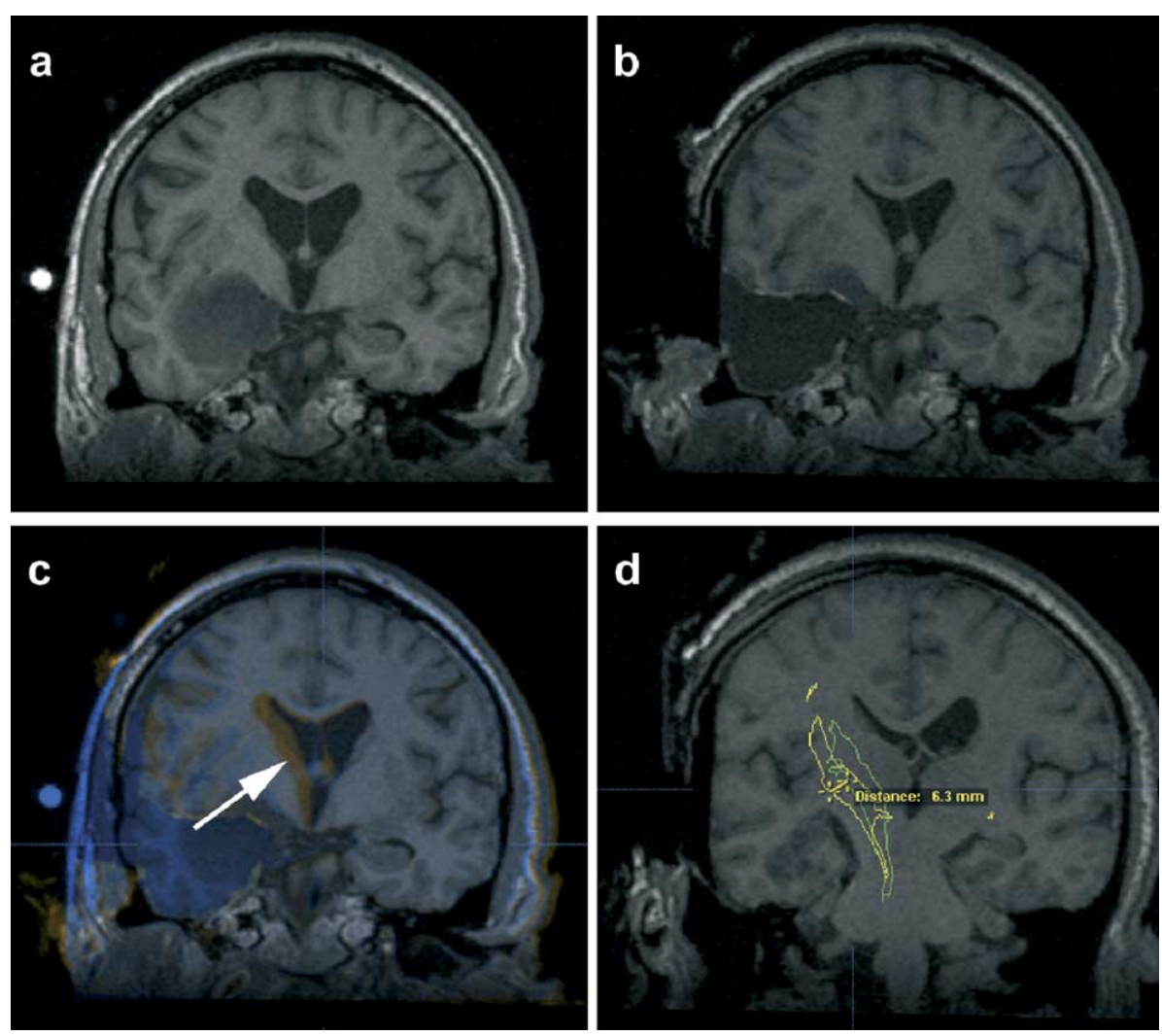

Fig. 5. Evaluation of shifting of major white matter tracts. Corresponding pre- and intraoperative images (a/b) are registered (c) (patient no. 5 with a right temporal WHO grade III astrocytoma). (c) Overlay of registered images; areas with extensive brain shift are depicted in yellow (white arrow). (d) Outlines of the pre- (yellow) and intraoperative (green) pyramidal tracts registered with the intraoperative images for evaluation of white matter tract shifting which amounts up to $-6 \mathrm{~mm}$ (inward shifting).

Concerning the accuracy of the delineation of the pyramidal tract, image registration and image distortion, as well as the patient registration error have to be taken into account. The image registration error amounted up to $3 \mathrm{~mm}$, which is in the range of integration of fMRI data into navigational datasets (Kober et al., 2001; Nimsky et al., 1999). The target registration error, as a measure for the patient registration error of $1.0 \pm 0.5 \mathrm{~mm}$, proved to have good clinical application accuracy. The $1.9 \times 1.9 \times 1.9 \mathrm{~mm}^{3}$ isotropic resolution of the applied DTI sequence is in the reported range necessary for an unambiguous identification of cortical activation in fMRI (Yoo et al., 2004). Echo planar images are distorted to a certain extent at the proximity of the skull base and near air-filled spaces, influencing the spatial accuracy e.g., in the middle and lower brainstem. Those artifacts can be attributed to the small bandwidth of the applied single shot echo planar imaging sequence in the phase-encoding direction and the inhomogeneity of the magnetic field due to a varying susceptibility within the measured tissue. We used a non-linear registration approach for an offline evaluation of the error caused by image distortion. This non-linear registration approach was too time-consuming so we could not use it for the integration of pre- or even intraoperative fiber tract data. Our strategy to define the coordinate system of the generated fiber tracts by a rigid registration of the $b=0$ diffusion images with anatomical 3-D data proved to be clinically applicable in the area of interest, i.e., at the deep margin of a tumor where the image distortion in relation to the spatial position of the pyramidal tract amounted up to only $3 \mathrm{~mm}$.

Adding all these errors in a worst case scenario would mean that a safety margin of about $5 \mathrm{~mm}$ when approaching the pyramidal tract should be taken into account. Furthermore, a potential, individually in its extent and direction, unpredictable shifting of major whiter matter tracts, that may exceed the extent of image distortion and registration error, has to be taken into account as well. Several studies on intraoperative imaging clearly revealed a major shifting of deep-seated tumor portions during tumor removal (Keles et al., 2003; Nabavi et al., 2001; Nimsky et al., 2000). Consequently, also functionally relevant structures, such as the pyramidal tract, are at risk of shifting, as first results on intraoperative diffusion-weighted imaging confirmed (Mamata et al., 2001). Intraoperative DTI revealed a marked shifting of white matter tracts during neurosurgical procedures (Nimsky et al., 2005a). As a consequence of this shifting, the preoperative functional data are no longer valid so that after a substantial removal of the tumor volume navigation can no longer be relied on, if this shifting is not compensated for. Therefore, it is necessary that not only intraoperative anatomical data are used to compensate for the effects of brain shift (Nimsky et al., 2000) but also functional data have to be updated. Mathematical models that describe the brain shift phenomenon using finite elements may be helpful in this respect (Ferrant et al., 2002). A logical step would be to use intraoperative DTI data and process them like we did for preoperative use for an intraoperative navigation updating. However, the whole data processing lasting about $20 \mathrm{~min}$ is still too timeconsuming so that it cannot yet be applied routinely during surgery. A promising approach, which we are working on, will be the integration of fiber tract generation directly in the navigation software, further reducing planning time, facilitating an intraoperative functional updating. 
Up to now, the combination of preoperative DTI data in combination with intraoperative cortical stimulation mapping (Berman et al., 2004) is a possibility to compensate for the effects of brain shift. However, when applying electrical subcortical mapping techniques, the subcortical pathways are only identified, when the resection margin is within these pathways or 2 to $3 \mathrm{~mm}$ away, leading to an additional motor deficit of up to $37 \%$ temporarily, decreasing to $7 \%$ permanent deficits. Furthermore, in $50 \%$, no subcortical pathways could be identified, complicating comparability (Keles et al., 2004). To compare the results of preoperative fiber tracking and intraoperative electrical stimulation (Kamada et al., 2005; Kinoshita et al., 2005), brain shift has to be taken into account, as well as all technical inaccuracies of the integration process of fiber tracking into navigational datasets. A practical solution not to underestimate the size of major white matter tracts (Kinoshita et al., 2005) and to avoid risking neurological deficits may be to add an additional hull to the 3-D object that represents the pyramidal tract in the navigation software. The thickness of this safety margin should ideally consider the quality of the initial data (e.g., in respect to image distortion), the quality of the registration process, as well as the extent of a potential shifting, if only preoperative fiber tract data are available.

Besides the aspects of spatial accuracy, limitations of our approach are related to the technique of fiber tracking per se. The choice of the different fiber tracking parameters like the accurate placement of seed regions, adjustment of the fiber step length, FA thresholds, etc. is a user-dependent process and is prone to errors. Clustering techniques of DTI data for extracting major white matter tracts may be a solution in this respect, and reliable pre-sets of tracking parameters have to be established when the proposed approach is applied on a broad clinical scale so that even nonspecialists, for example, will be able to delineate the course of the pyramidal tract reliably. Further limitations relate to the tracking algorithm itself and the actual data acquisition so that, for example, areas of fiber crossings are not considered by the current implementation. However, when focusing on the delineation of the pyramidal tract in a clinical setting, areas of fiber crossings are not of major relevance.

Of course, the number of investigated patients in the current study is too small to generalize the effects of the described method on clinical outcome. The aim of the study was to demonstrate the feasibility of our approach to integrate fiber tract data into a navigational setup. As integration of functional data from fMRI or MEG identifying the motor-cortex- or language-related areas has resulted in less morbidity (Ganslandt et al., 1999), it is plausible that the additional information gained by integrated localization of major white matter tracts, namely, the pyramidal tract, will further enhance surgical safety (Wu et al., 2003). The clinical impact of integrating information on major white matter tracts into navigational setups may be emphasized by summarizing our clinical data from this study with our previous one where we localized the pyramidal tract by a rough segmentation of color-encoded FA maps which were then registered with 3-D navigational data (Nimsky et al., 2005c). In total, in both studies, 35 patients with gliomas were operated and only in one patient, i.e., $2.9 \%$, we observed a new postoperative neurological deficit. We think that integrating the fiber tract data into a navigation system so that they can be visualized in the surgical field provides the most intuitive possibility for the neurosurgeon to know the actual course of the pyramidal tract during surgery so that its function can be best preserved. The visualization of fiber tracts on a screen besides the surgical site, even depicting their actual intraoperative position, as we have implemented with intraoperative fiber tracking (Nimsky et al., 2005b), gives only a clue about their actual position in the surgical field. Investigating larger patient series to study the clinical effects of integrating fiber tract data into the navigation on patient outcome is one of the next major steps.

Sophisticated functional imaging defines nearly all parts of the brain as eloquent areas so that at the end there may be no noneloquent zone for the neurosurgeon to enter the brain and to remove a tumor. Integration of fiber tract data into navigational datasets is part of this process. The application of such a technique has not only the benefit to potentially decrease the risk of postoperative neurological deficits; it may also result in some kind of under-resection of tumors just to avoid postoperative deficits. In this study, we achieved a gross total resection only in 6 of the 17 patients undergoing resection, this may support this assumption. We apply intraoperative imaging to maximize the extent of resection, which on the other hand is minimized by our aim to prevent postoperative neurological deficits. However, without having the implemented technique available, some of our patients would not at all have been scheduled for a resection of their tumor. Despite decreasing non-eloquent space for resection, it is out of doubt that today tumors are operated, which we thought we should not operate on only 10 years ago.

\section{Acknowledgments}

We express our special thanks to Edgar Müller, PhD, Theodor Vetter, PhD, and Michael Zwanger, PhD (Siemens Medical Solutions, Erlangen, Germany) for their continuous technical advice, as well as to Stefanie Kreckel, RT, for her technical support in MRI. Furthermore, we acknowledge valuable support by Ruopeng Wang (Department of Radiology/Nuclear Magnetic Resonance Center, Massachusetts General Hospital, Boston, USA) for programming the DTI taskcard software, as well as U. Mezger (BrainLab, Heimstetten, Germany) for programming the fiber tract import routine for the navigation software. This work was supported in part by the Deutsche Forschungsgemeinschaft in the context of project C9 of SFB603.

\section{References}

Basser, P.J., Mattiello, J., LeBihan, D., 1994. MR diffusion tensor spectroscopy and imaging. Biophys. J. 66, 259-267.

Basser, P.J., Pajevic, S., Pierpaoli, C., Duda, J., Aldroubi, A., 2000. In vivo fiber tractography using DT-MRI data. Magn. Reson. Med. 44, $625-632$

Beppu, T., Inoue, T., Shibata, Y., Kurose, A., Arai, H., Ogasawara, K., Ogawa, A., Nakamura, S., Kabasawa, H., 2003. Measurement of fractional anisotropy using diffusion tensor MRI in supratentorial astrocytic tumors. J. Neuro-Oncol. 63, 109-116.

Berman, J.I., Berger, M.S., Mukherjee, P., Henry, R.G., 2004. Diffusiontensor imaging-guided tracking of fibers of the pyramidal tract combined with intraoperative cortical stimulation mapping in patients with gliomas. J. Neurosurg. 101, 66-72.

Clark, C.A., Barrick, T.R., Murphy, M.M., Bell, B.A., 2003. White matter fiber tracking in patients with space-occupying lesions of the brain: a new technique for neurosurgical planning? NeuroImage 20, 1601-1608.

Coenen, V.A., Krings, T., Mayfrank, L., Polin, R.S., Reinges, M.H., Thron, 
A., Gilsbach, J.M., 2001. Three-dimensional visualization of the pyramidal tract in a neuronavigation system during brain tumor surgery: first experiences and technical note. Neurosurgery 49, 86-93.

Duffau, H., Capelle, L., Denvil, D., Sichez, N., Gatignol, P., Taillandier, L., Lopes, M., Mitchell, M.C., Roche, S., Muller, J.C., Bitar, A., Sichez, J.P., van Effenterre, R., 2003. Usefulness of intraoperative electrical subcortical mapping during surgery for low-grade gliomas located within eloquent brain regions: functional results in a consecutive series of 103 patients. J. Neurosurg. 98, 764-778.

Ferrant, M., Nabavi, A., Macq, B., Black, P.M., Jolesz, F.A., Kikinis, R., Warfield, S.K., 2002. Serial registration of intraoperative MR images of the brain. Med. Image Anal. 6, 337-359.

Ganslandt, O., Fahlbusch, R., Nimsky, C., Kober, H., Möller, M., Steinmeier, R., Romstöck, J., Vieth, J., 1999. Functional neuronavigation with magnetoencephalography: outcome in 50 patients with lesions around the motor cortex. J. Neurosurg. 91, 73-79.

Guye, M., Parker, G.J., Symms, M., Boulby, P., Wheeler-Kingshott, C.A., Salek-Haddadi, A., Barker, G.J., Duncan, J.S., 2003. Combined functional MRI and tractography to demonstrate the connectivity of the human primary motor cortex in vivo. NeuroImage 19, 1349-1360.

Hendler, T., Pianka, P., Sigal, M., Kafri, M., Ben-Bashat, D., Constantini, S., Graif, M., Fried, I., Assaf, Y., 2003. Delineating gray and white matter involvement in brain lesions: three-dimensional alignment of functional magnetic resonance and diffusion-tensor imaging. J. Neurosurg. 99, 1018-1027.

Holodny, A.I., Schwartz, T.H., Ollenschleger, M., Liu, W.C., Schulder, M., 2001. Tumor involvement of the corticospinal tract: diffusion magnetic resonance tractography with intraoperative correlation. J. Neurosurg. 95, 1082 .

Inoue, T., Shimizu, H., Yoshimoto, T., 1999. Imaging the pyramidal tract in patients with brain tumors. Clin. Neurol. Neurosurg. 101, 4-10.

Kamada, K., Houkin, K., Takeuchi, F., Ishii, N., Ikeda, J., Sawamura, Y., Kuriki, S., Kawaguchi, H., Iwasaki, Y., 2003. Visualization of the eloquent motor system by integration of MEG, functional, and anisotropic diffusion-weighted MRI in functional neuronavigation. Surg. Neurol. 59, 352-362.

Kamada, K., Todo, T., Masutani, Y., Aoki, S., Ino, K., Takano, T., Kirino, T., Kawahara, N., Morita, A., 2005. Combined use of tractographyintegrated functional neuronavigation and direct fiber stimulation. J. Neurosurg. 102, 664-672.

Keles, G.E., Lamborn, K.R., Berger, M.S., 2003. Coregistration accuracy and detection of brain shift using intraoperative sononavigation during resection of hemispheric tumors. Neurosurgery 53, 556-564.

Keles, G.E., Lundin, D.A., Lamborn, K.R., Chang, E.F., Ojemann, G., Berger, M.S., 2004. Intraoperative subcortical stimulation mapping for hemispherical perirolandic gliomas located within or adjacent to the descending motor pathways: evaluation of morbidity and assessment of functional outcome in 294 patients. J. Neurosurg. 100, 369-375.

Kinoshita, M., Yamada, K., Hashimoto, N., Kato, A., Izumoto, S., Baba, T., Maruno, M., Nishimura, T., Yoshimine, T., 2005. Fiber-tracking does not accurately estimate size of fiber bundle in pathological condition: initial neurosurgical experience using neuronavigation and subcortical white matter stimulation. NeuroImage 25, 424-429.

Kober, H., Nimsky, C., Möller, M., Hastreiter, P., Fahlbusch, R., Ganslandt, O., 2001. Correlation of sensorimotor activation with functional magnetic resonance imaging and magnetoencephalography in presurgical functional imaging: a spatial analysis. NeuroImage 14, $1214-1228$.

Krings, T., Reinges, M.H., Thiex, R., Gilsbach, J.M., Thron, A., 2001. Functional and diffusion-weighted magnetic resonance images of spaceoccupying lesions affecting the motor system: imaging the motor cortex and pyramidal tracts. J. Neurosurg. 95, 816-824.

Le Bihan, D., Van Zijl, P., 2002. From the diffusion coefficient to the diffusion tensor. NMR Biomed. 15, 431-434.

Lu, S., Ahn, D., Johnson, G., Cha, S., 2003. Peritumoral diffusion tensor imaging of high-grade gliomas and metastatic brain tumors. Am. J. Neuroradiol. 24, 937-941.
Mamata, Y., Mamata, H., Nabavi, A., Kacher, D.F., Pergolizzi Jr., R.S., Schwartz, R.B., Kikinis, R., Jolesz, F.A., Maier, S.E., 2001. Intraoperative diffusion imaging on a 0.5 Tesla interventional scanner. J. Magn. Reson. Imaging 13, 115-119.

Merhof, D., Hastreiter, P., Soza, G., Stamminger, M., Nimsky, C., 2004. Non-linear integration of DTI-based fiber tracts into standard 3D MR data. In: Girod, B., Magnor, M., Seidel, H. (Eds.), Vision Modeling and Visualization. Akademische Verlagsgesellschaft Aka, Berlin, pp. 371-377.

Mori, S., Van Zijl, P.C., 2002. Fiber tracking: principles and strategies-A technical review. NMR Biomed. 15, 468-480.

Mori, S., Crain, B.J., Chacko, V.P., van Zijl, P.C., 1999. Three-dimensional tracking of axonal projections in the brain by magnetic resonance imaging. Ann. Neurol. 45, 265-269.

Nabavi, A., Black, P.M., Gering, D.T., Westin, C.F., Mehta, V., Pergolizzi Jr., R.S., Ferrant, M., Warfield, S.K., Hata, N., Schwartz, R.B., Wells III, W.M., Kikinis, R., Jolesz, F.A., 2001. Serial intraoperative magnetic resonance imaging of brain shift. Neurosurgery 48, 787-798.

Nimsky, C., Ganslandt, O., Kober, H., Möller, M., Ulmer, S., Tomandl, B., Fahlbusch, R., 1999. Integration of functional magnetic resonance imaging supported by magnetoencephalography in functional neuronavigation. Neurosurgery 44, 1249-1256.

Nimsky, C., Ganslandt, O., Cerny, S., Hastreiter, P., Greiner, G., Fahlbusch, R., 2000. Quantification of, visualization of, and compensation for brain shift using intraoperative magnetic resonance imaging. Neurosurgery 47, $1070-1080$.

Nimsky, C., Ganslandt, O., Keller v, B., Romstöck, J., Fahlbusch, R., 2004. Intraoperative high-field strength MR imaging: implementation and experience in 200 patients. Radiology 233, 67-78.

Nimsky, C., Ganslandt, O., Hastreiter, P., Wang, R., Benner, T., Sorensen, A.G., Fahlbusch, R., 2005a. Intraoperative diffusion tensor imaging: shifting of white matter tracts during neurosurgical procedures-Initial experience. Radiology 234, 218-225.

Nimsky, C., Ganslandt, O., Hastreiter, P., Wang, R., Benner, T., Sorensen, A.G., Fahlbusch, R., 2005b. Preoperative and intraoperative diffusion tensor imaging-based fiber tracking in glioma surgery. Neurosurgery 56 , $130-138$.

Nimsky, C., Grummich, P., Sorensen, A.G., Fahlbusch, R., Ganslandt, O., 2005c. Visualization of the pyramidal tract in glioma surgery by integrating diffusion tensor imaging in functional neuronavigation. Zentralbl. Neurochir. 66, 133-141.

Pajevic, S., Pierpaoli, C., 1999. Color schemes to represent the orientation of anisotropic tissues from diffusion tensor data: application to white matter fiber tract mapping in the human brain. Magn. Reson. Med. 42, $526-540$.

Price, S., Burnet, N., Donovan, T., Green, H., Pena, A., Antoun, N., Pickard, J., Carpenter, T., Gillard, J., 2003. Diffusion tensor imaging of brain tumours at $3 \mathrm{~T}$ : a potential tool for assessing white matter tract invasion? Clin. Radiol. 58, 455-462.

Romstöck, J., Fahlbusch, R., Ganslandt, O., Nimsky, C., Strauss, C., 2002. Localisation of the sensorimotor cortex during surgery for brain tumors: feasibility and waveform patterns of somatosensory evoked potentials. J. Neurol., Neurosurg. Psychiatry 72, 221-229.

Stieltjes, B., Kaufmann, W.E., van Zijl, P.C., Fredericksen, K., Pearlson, G.D., Solaiyappan, M., Mori, S., 2001. Diffusion tensor imaging and axonal tracking in the human brainstem. NeuroImage 14, 723-735.

Studholme, C., Hill, D., Hawkes, D., 1996. Automated 3D registration of MR and CT images of the head. Med. Image Anal. 1, 163-175.

Talos, I., O’Donnell, L., Westin, C.F., Warfield, S.K., Wells III, W.M., Yoo, S., Panych, L., Golby, A., Mamata, H., Maier, S., Ratiu, P., Guttmann, C., Black, P.M., Jolesz, F., Kikinis, R., 2003. Diffusion tensor and functional MRI fusion with anatomical MRI for image-guided neurosurgery. In: Ellis, R., Peters, T. (Eds.), MICCAI 2003. Springer-Verlag, Berlin, Heidelberg, pp. 407-415.

Thevenaz, P., Unser, M., 1996. A pyramid approach to sub-pixel image fusion based on mutual information. Proc. IEEE Int. Conf. Image Process. 1, 265-268. 
Tummala, R.P., Chu, R.M., Liu, H., Truwit, C.L., Hall, W.A., 2003. Application of diffusion tensor imaging to magnetic-resonance-guided brain tumor resection. Pediatr. Neurosurg. 39, 39-43.

Wieshmann, U.C., Clark, C.A., Symms, M.R., Franconi, F., Barker, G.J., Shorvon, S.D., 1999. Reduced anisotropy of water diffusion in structural cerebral abnormalities demonstrated with diffusion tensor imaging. Magn. Reson. Imaging 17, 1269-1274.

Witwer, B.P., Moftakhar, R., Hasan, K.M., Deshmukh, P., Haughton, V., Field, A., Arfanakis, K., Noyes, J., Moritz, C.H., Meyerand, M.E., Rowley, H.A., Alexander, A.L., Badie, B., 2002. Diffusion-tensor imaging of white matter tracts in patients with cerebral neoplasm. J. Neurosurg. 97, 568-575.

Wu, J.S., Zhou, L.F., Hong, X.N., Mao, Y., Du, G.H., 2003. Role of diffusion tensor imaging in neuronavigation surgery of brain tumors involving pyramidal tracts. Zhonghua Waike Zazhi 41, 662-666.

Yamada, K., Kizu, O., Mori, S., Ito, H., Nakamura, H., Yuen, S., Kubota, T., Tanaka, O., Akada, W., Sasajima, H., Mineura, K., Nishimura, T., 2003. Brain fiber tracking with clinically feasible diffusion-tensor MR imaging: initial experience. Radiology 227, 295-301.

Yingling, C.D., Ojemann, S., Dodson, B., Harrington, M.J., Berger, M.S., 1999. Identification of motor pathways during tumor surgery facilitated by multichannel electromyographic recording. J. Neurosurg. 91, 922-927.

Yoo, S.S., Talos, I.F., Golby, A.J., Black, P.M., Panych, L.P., 2004. Evaluating requirements for spatial resolution of fMRI for neurosurgical planning. Hum. Brain Mapp. 21, 34-43. 